\title{
Atherogenic Index of Plasma among Type2 Diabetic Patients Cross-Sectional Study In Iraq
}

\author{
Dalal Abd Al-Sattar Asaad ${ }^{1}$, Ali Shalash Sultan ${ }^{1}$, Zaid Naji Hassan ${ }^{1}$ \\ ${ }^{1}$ Biology Department, College of Science-Mustansiriyah University/Iraq
}

\begin{abstract}
The study was carried out on (60) males previously diagnosed with diabetes mellitus type 2 aged (40$55)$ years with mean \pm SE of age $(48.46 \pm 5.03)$, who visited the Specialist Center for Endocrine and Diabetes at Baghdad province.The total cholesterol(TC) $\mathrm{mg} / \mathrm{dl}$ levels were $(180.90 \pm 11.68,209.60 \pm 14.50$ ,203.10 \pm 14.65$)$ respectively showed non significant $(\mathrm{p}>0.05)$ variation in diabetic groups ,TG $\mathrm{mg} / \mathrm{dl}$ levels in normal weight group $(172.55 \pm 14.13)$ decreased significantly $(\mathrm{p}<0.01)$ in comparison with over weight and obese $(273.95 \pm 22.83) ;(306.95 \pm 25.20)$,respectively. LDL mg/dl levels were (101.50 \pm 9.56 ; $110.00 \pm 12.71 ; 105.40 \pm 9.99)$ respectively showed non significant $(p>0.05)$ variation in diabetic groups. VLDL $\mathrm{mg} / \mathrm{dl}$ in normal weight group $(35.75 \pm 3.31)$ decreased significantly $(\mathrm{p}<0.01)$ in comparison with over weight and obese (59.70 \pm 4.59$)$; (61.30 \pm 5.03$) . \mathrm{HDL} \mathrm{mg} / \mathrm{dl}$ levels were $(43.20 \pm 2.01,42.85 \pm 3.05,40.05 \pm 3.32)$ respectively showed non significant $(\mathrm{p}>0.05)$ variation in diabetic groups. FBG showed non-significant correlation with TC( $\mathrm{r}=-0.13), \mathrm{LDL}(\mathrm{r}=-0.13), \mathrm{TG}(\mathrm{r}=-0.12), \mathrm{VLDL}(\mathrm{r}=-0.15), \mathrm{HDL}(\mathrm{r}=0.24)$. HbA1C showed positive significant $(\mathrm{p}<0.05)$ correlation with $\mathrm{TC}(\mathrm{r}=0.25)$, while showed non significant correlation with TG $(r=0.10)$,LDL $(r=0.18)$,VLDL $(r=-0.01)$, HDL $(r=0.16)$. BMI showed non-significant with $\mathrm{TC}(\mathrm{r}=0.15), \mathrm{LDL}(\mathrm{r}=0.08)$, HDL, $(\mathrm{r}=-0.08)$. While positive significant $(\mathrm{p}<0.01)$, correlation with $\mathrm{TG}(\mathrm{r}=0.50)$, $\operatorname{VLDL}(\mathrm{r}=0.39)$. The levels of AIP differ significantly $(\mathrm{p}<0.01)$ among diabetic groups $(0.576 \pm 0.04 ; 0.800 \pm$ 0.06 and $0.911 \pm 0.05)$ but over weight and obese $(0.800 \pm 0.06 ; 0.911 \pm 0.05)$ were not significant $(\mathrm{p}>0.05)$. AIP was significantly correlated with body mass index $(r=0.25)$, visceral fat $(r=0.37)$, TC $(r=0.22)$, LDL $(\mathrm{r}=0.24), \mathrm{HDL}(\mathrm{r}=-0.72), \mathrm{TG}(\mathrm{r}=0.84)$, glucose $(\mathrm{r}=0.32)$. Positive correlation $(\mathrm{P}<0.01)$ with $\mathrm{BMI}(\mathrm{r}=0.36)$ ;VLDL $(\mathrm{r}=0.28)(\mathrm{p}<0.05)$ and negative correlation $(\mathrm{p}<0.01)$ with HDL-C $(\mathrm{r}=-0.32)$ was seen .
\end{abstract}

Keywords:- Diabetes mellitus ,Dyslipidemia,lipid profile,Atherogenic index of plasma(AIP).Isulin resistance(IR).

\section{Introduction}

Dyslipidemia in T2DM is characterized by high level Of total cholestrol (TC), low density lipoprotein (LDL-C), triglyceride (TG) with low level of (HDL-C) ${ }^{1}$.The early detection of lipid abnormalities with their treatment can reduce the risk of atherogenic cardiovascular and cerebrovascular disorders in T2DM patients ${ }^{2}$.Atherosclerotic coronary artery disease and different form of cardiovascular disease (CVA) are the principle causing of morbidity and mortality in T2DM, that alterations in lipid profile lead to atherosclerosis in $\mathrm{T}^{2} \mathrm{DM}^{3}$. The deffintion of atherogenic index of plasma AIP is used logaritham $(\log )$ of the ratio of plasma concentration of TG/HDL-C. Which was correlated with size of HDLand LDL molcules ${ }^{4}$. The AIP is regarded as astrong marker for plasma atherogenicity and correlated with (CVD) .The AIP value under 0.11 is associated with low risk of CVD but value between 0.11-0.21 and above than 0.21 causes intermediate and elevated risk respectively ${ }^{5}$. Diabetes (T2DM) and related cardiovascular complication are major public health challenges among world wide. Individuals with T2DM have two to four fold risk of coronary artery disease (CAD) causing death among people of Type2Diabetes mellitus ${ }^{6}$. Dyslipidemia participates in causing of atherosclerosis, which pathological affects of CVD persons with metabolic syndrome or T2DM exhibit a characterstics pattern of serum lipid abnormalities, consisting of low HDL-C and increased TG levels with increased LDL particles ${ }^{7}$.Various mechanisms forming the atherogenic lipid disorders in Type 2 patients. The increase in breakdown of 
intracellular Triglycerides and release of Free fatty acids increased into the blood causing free fatty acids hepatic infiltration, muscle, pancreatic B-cell.predisposing to T2DM .Increased hepatic FFAS contribute to elevation of hepatic TG with subsequently resulting in increased VLDL particles. From other side cholesterol ester transfer protein (CETP) converts Triglycerides from Very low density lipoprotein to cholesterol found in HDL and LDL resulting to cholesterol rich atherogenic VLDL. particles.HDLparticles that under go these modification are cleared the renal function,resulting in decrease HDL-C levels ${ }^{8}$. Endothelial dysfunction (ED) is another major factor for the development of atherosclerosis.It might may be caused by dyslipidemia ,oxidative stress, inflammation,lipid abnormality regard the major cause of ED,espically LDL particles are more susceptible to oxidation, oxidized LDL particles reduces the availability of nitric oxide (NO) and impair endothelial function. This creates appositive feedback loop in which inflammatory factors promote monocyte and Tcell adhesion, foam cell information,extracellular matix digestion and vascular smooth muscle migration and proliferation leading to atherosclerosis ${ }^{9}$.The prostacyclin and Nitric oxide production by normal endothelial inhibition with platelet reactivation and relaxation vascular smooth muscle improving circulation influx normaly. Diabetic patients showed reduction in release of prostacyclin and Nitric oxide with chronic defects of endothelial Nitic oxide synthesis action, which is a predictor of dyslipidemia and explain partly the accelerated atherosclerosis in $\mathrm{DM}^{10}$. In diabetes long term hyperglycemia causes generalized vascular endothelial damage which reduced function of lipoprotein lipase leading to increase TG,TC, and LDL ${ }^{11}$.

\section{Material and Method}

\section{Collection of Information and Patients Selection}

The study was carried out on (60) males previously diagnosed with diabetes mellitus type2 aged (40$55)$ years with mean \pm SE of age $(48.46 \pm 5.03)$, who visited the Specialist Center for Endocrine and Diabetes at Baghdad province.The study was begun from 1/ November 2018 to 30/January 2019. Informed consent was obtained from patients cross sectional study to fill the questionnaire form them before venipuncture which was used for recording the necessary information that concerning with subject groups which included name, age, length, body weight, and duration of diabetes onset.

\section{Study design:-}

The subject study included group (60) patients as diabetic group (cross sectional study). The diabetic group was divided into three subgroups according to BMI

Normal weight BMI (18.5-24.9) Kg/m².

Over weight (OV) BMI (25-29.9) Kg/m².

Obese BMI $(\leq 30) \mathrm{Kg} / \mathrm{m}^{2}$.

\section{2-Collection of blood samples:-}

Eight milliliters of blood were drawn from each patient after (12-14) hours fasting via venipuncture, by using $10 \mathrm{ml}$ disposable syringes between $(8.00-10.30$ A.M). Blood specimen used in two aliquots; two and three $\mathrm{ml}$. The first one was put in a tube have ethylene diamine tetra acetic acid (EDTA K3) as anticoagulant and stored at $\left(2-8^{\circ} \mathrm{C}\right)$ for analyses of $\mathrm{HbA} 1 \mathrm{c}$, while the second aliquot was transferred into gel tubes without anti-coagulant; blood was left to clot for 20-30 minutes at $\left(37^{\circ} \mathrm{C}\right)$ in an incubator. Serum were separated by centrifugation at $3000 \mathrm{rpm}$ for 10 minutes used for the determination of FBG, lipids profile, serum was stored in Eppendorf tubes at $\left(-20^{\circ} \mathrm{C}\right)$ for analyses later.

\section{Lipid profile calculation:-}

Measurement of serum total cholesterol (TC) Cholesterol was determined by using the Mindray Bs-200 12. Measurement of Triglycerides (TG) Concentration. Triglyceride was determined by using the Kit SPINREACT according to the method by mindary 12. Measurement of Serum High Density Lipoprotein (HDL) Concentration. MINDRAY BS-120 / 200E Autoanalyzer use SPINREACT kit ${ }^{13}$. Measurement of Low Density Lipoprotein (LDL).Mindray BS-120/ 200E Autoanalyzer use SPINREACT kit ${ }^{13}$. VLDL(mg/ $\mathrm{dl})=$

Atherogenic index of plasma was measured using base 10 logarithm of the ratio Triglycerides to HDL, formula $=\log (\mathrm{TG} / \mathrm{HDL}-\mathrm{C})$. (Dobiásová and Frohlich ,2001).AIP $=\mathbf{L o g}$. 


\section{Results and Discussion}

Table (1) Effect of different groups of diabetic subjects in lipid profile.(Mean $\pm \mathrm{SE}$ )

\begin{tabular}{|c|c|c|c|c|}
\hline \multirow{2}{*}{$\begin{array}{l}\text { Parameters } \\
(\mathrm{mg} / \mathrm{dl})\end{array}$} & \multicolumn{3}{|l|}{ Groups } & \multirow{2}{*}{ LSD value } \\
\hline & Normal weight & Over weight & Obese & \\
\hline Total cholesterol & $180.90 \pm 11.68 \mathrm{a}$ & $209.60 \pm 14.50 \mathrm{a}$ & $203.10 \pm 14.65 \mathrm{a}$ & $38.747 \mathrm{NS}$ \\
\hline Triglyceride & $172.55 \pm 14.13 b$ & $273.95 \pm 22.83 \mathrm{a}$ & $306.95 \pm 25.20 \mathrm{a}$ & $60.221 * *$ \\
\hline LDL & $101.50 \pm 9.56 \mathrm{a}$ & $110.00 \pm 12.71 \mathrm{a}$ & $105.40 \pm 9.99 \mathrm{a}$ & $30.722 \mathrm{NS}$ \\
\hline VLDL & $35.75 \pm 3.31 b$ & $59.70 \pm 4.59 \mathrm{a}$ & $61.30 \pm 5.03 \mathrm{a}$ & $12.390 * *$ \\
\hline HDL & $43.20 \pm 2.01 \mathrm{a}$ & $42.85 \pm 3.05 \mathrm{a}$ & $40.05 \pm 3.32 \mathrm{a}$ & $8.081 \mathrm{NS}$ \\
\hline \multicolumn{5}{|c|}{$* *(\mathrm{P}<0.01), \mathrm{NS}:$ Non-Significant. } \\
\hline
\end{tabular}

From the result table (1), the TC mg/dl levels were (1 $80.90 \pm 11.68,209.60 \pm 14.50,203.10 \pm 14.65)$ respectively showed non significant $(\mathrm{p}>0.05)$ variation in diabetic groups ,TG $\mathrm{mg} / \mathrm{dl}$ levels in normal weight group $(172.55 \pm 14.13)$ decreased significantly $(p<0.01)$ in comparison with over weight and obese (273.95 \pm 22.83$)$ ;(306.95 \pm 25.20$)$.respectively. LDL $\mathrm{mg} / \mathrm{dl}$ levels were
$(101.50 \pm 9.56 ; 110.00 \pm 12.71 ; 105.40 \pm 9.99)$ respectively showed non significant $(\mathrm{p}>0.05)$ variation in diabetic groups.VLDL mg/dlinnormal weight group $(35.75 \pm 3.31)$ decreased significantly $(\mathrm{p}<0.01)$ in comparison with over weight and obese $(59.70 \pm 4.59)$; $(61.30 \pm 5.03)$. HDL $\mathrm{mg} / \mathrm{dl}$ levels were $(43.20 \pm 2.01,42.85 \pm 3.05,40.05 \pm 3.32)$ respectively showed non significant $(\mathrm{p}>0.05)$ variation in diabetic groups.

Table (2) Correlation coefficient between lipid profile with FBG,

HbA1c and BMI in diabetic patients.

\begin{tabular}{|l|l|l|l|}
\hline \multirow{2}{*}{ Parameters } & \multicolumn{2}{l|}{ Correlation coefficient-r } \\
\cline { 2 - 4 } & FBG & HbA1c & BMI \\
\hline Total cholesterol & $-0.13 \mathrm{NS}$ & $0.25^{*}$ & $0.15 \mathrm{NS}$ \\
\hline Triglyceride & $-0.12 \mathrm{NS}$ & $0.10 \mathrm{NS}$ & $0.50 * *$ \\
\hline LDL & $-0.13 \mathrm{NS}$ & $0.18 \mathrm{NS}$ & $0.08 \mathrm{NS}$ \\
\hline VLDL & $-0.15 \mathrm{NS}$ & $-0.01 \mathrm{NS}$ & $0.39 * *$ \\
\hline HDL & $0.24 \mathrm{NS}$ & $0.16 \mathrm{NS}$ & $-0.08 \mathrm{NS}$ \\
\hline$*(\mathrm{P}<0.05), * *(\mathrm{P}<0.01)$, NS: Non-Significant. & & \\
\hline
\end{tabular}

From the result table (2), FBG showed nonsignificant link with $\mathrm{TC}(\mathrm{r}=-0.13), \mathrm{LDL}(\mathrm{r}=-0.13), \mathrm{TG}(\mathrm{r}=-$ $0.12), \operatorname{VLDL}(\mathrm{r}=-0.15), \mathrm{HDL}(\mathrm{r}=0.24)$. HbA1C showed positive significantly $(\mathrm{p}<0.05)$ correlated with $\mathrm{TC}(\mathrm{r}=$
$0.25)$, and showed non significant correlation with TG $(\mathrm{r}=0.10), \operatorname{LDL}(\mathrm{r}=0.18), \operatorname{VLDL}(\mathrm{r}=-0.01), \operatorname{HDL}(\mathrm{r}=0.16)$. BMI showed non-significant with TC $(\mathrm{r}=0.15)$, LDL $(\mathrm{r}=0.08)$, HDL $(\mathrm{r}=-0.08)$. positive significantly $(\mathrm{p}<0.01)$ 
correlated with TG $(r=0.50), \operatorname{VLDL}(r=0.39)$. The dyslipidemia means to change in lipid profile involving elevating serum TC, TG, decreased HDL, elevation LDL and VLDL. Dyslipidemia is present to be accompany with both, insulin resistance and coronary heart disease ${ }^{8}$.Studies lipids profile in T2DM and control subjects in overweight and obese groups in Saudi Arabia was seen high incidence of dyslipidemia in T2DM patients ( Our results showed non significant increase of TC in overweight and obese diabetic patient $>200 \mathrm{mg}$ and significantly higher TG and VLDL levels; while LDL and HDL levels were non-significant this attributed to that excess acetyl CoA from lipolysis of fat to free fatty acids (FFA) gets converted to more and more cholesterol and its concentration in blood rises in T2DM.VLDL increased either because of increased hepatic production of VLDL or decreased removal of VLDL circulation. Serum concentration of TG also increases because of decreased removal from circulation. The increased TG in our study was agreed with (Taskinen,2003). who reported the increased serum TG levels in T2DM patients are widley because of an elevation VLDL, specially large VLDL1 molcules.Both rising production and late catabolism of VLDL are responsible for the rising VLDL.Insulin resistance is accompanied with reduction activity of hormone-sensitive lipase in adipose tissue by insulin, resulting lipolysis increasing and, portal flux of NEFA to hepatic cells to stimulate synthesis of TG in hepatic cellsThe role of increasing in TG levels in hyperglycemic patients also includes decrease of lipoprotein lipase (LPL) activity. It has been recorded that LPL activity is reduced in patients with T2DM.( Nikkila and Taskinen,1981).LPL hydrolyses TG of chylomicrons and (VLDL). The FFA flux into glycogen rich hepatocytes stimulates TG synthesis, which produced of VLDL-cholesterol..Obesity and T2DM are accompanied by elevation deposition of TG in non-adipose tissue, like heart, liver, pancreas, and skeletal muscle. Obesity, fat accumulated in the under skin, abdominal and visceral depots, which is have affinity in risk of metabolic and heart blood vessels complications; Obesity is positively affecting risk the developing of T2DM,dyslipidemia, IR and hypertensive. It characterized by adipose tissue defects growth resulting in increasing size of fat cell (hypertrophic obesity) or fat cell number (hyperplastic obesity), or both. Obesity is known as body mass index (BMI). As we observed mean serum TG levels higher in diabetics groups .As BMI showed high significant correlation with $\mathrm{TG}(\mathrm{r}=0.50)$ and VLDL $(\mathrm{r}=0.39)$. Higher HbA1c concentrations in our study were associated significantly correlation with higher $\mathrm{TC}(\mathrm{r}=0.25) \quad(\mathrm{P}<0.05$ reported that significant linear positive correlation with $\mathrm{HbA1c}$ and lipid parameters except HDL in T2DM group.

Table (3) Effect of different groups in Atherogenic index of plasma

(AIP) in diabetic patients.

\begin{tabular}{|l|l|}
\hline Groups & Mean \pm SE of AIP \\
\hline Normal weight & $0.576 \pm 0.04 \mathrm{~b}$ \\
\hline Over weight & $0.800 \pm 0.06 \mathrm{a}$ \\
\hline Obese & $0.911 \pm 0.05 \mathrm{a}$ \\
\hline LSD value & $0.154 * *$ \\
\hline$* *(\mathrm{P}<0.01)$. & \\
\hline
\end{tabular}

The levels of AIP differ significantly $(\mathrm{p}<0.01)$ among diabetic groups $(0.576 \pm 0.04 ; 0.800 \pm 0.06$ and $0.911 \pm 0.05)$ but over weight and obese $(0.800 \pm 0.06$; $0.911 \pm 0.05)$ were not significant $(\mathrm{p}>0.05)$, table $(12-3)$, Figure 1.

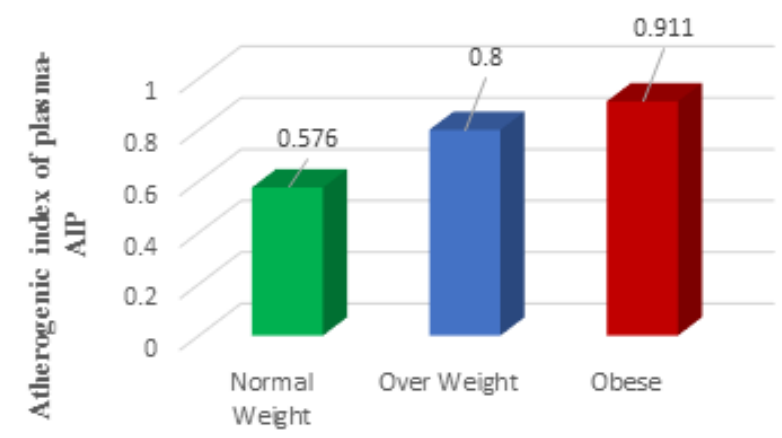

Figure 1 showing Atherogenic index among diabetic patients

AIP values were above normal values (under 0.11 ) while 0.11 and 0.21 values of AIP associated with intermediate and high risk of CVD $\left({ }^{5}\right)$. Previous researchers they concluded that AIP was elevated in diabetic and neuro diabetic and could be used a marker for predicting CVD in patients groups. AIP showed the presence of atherogenic minute LDL and HDL molcules, is a marker prediction of heart atherosclerosis and cardiovascular dangerous as an alternative for IR. ${ }^{4,14}$ The AIP was significantly higher in 300 T2DM patients in Bangladesh and revealed dyslipidemia with increased TG and decreased serum HDL-C levels . AIP showed correlation BMI $(r=0.25)$, visceral fat $(r=0.37)$, 
TC $(r=0.22)$, LDL $(r=0.24)$, HDL $(r=-0.72)$, TG $(r=$ $0.84)$, glucose $(r=0.32)$.. Other Researchers the found TG to HDL was a strong prediction of heart infarction .That atherogenic index of plasma was an index of highest sensitivity for predicting the acute coronary events . That AIP correlation with BMI $(\mathrm{r}=0.182, \mathrm{P}<$ 0 001), (FBG $(r=0.153)$. AIP is used for detection of Type 2Diabetic Mellitus with high risk of complications 15.

\section{Conclusions}

AIP is used clinically for prediction of Type Diabetic Mellitus with higher risk of complications and this correlated with lipid profile,BMI .

Acknowledgement:-The authors would like to thank mustansiriyah university (www.uomustansiryiah .edu.iq)Baghdad-Iraq for its support in the present work .

Ethical Clearance: The Research Ethical Committee at scientific research by ethical approval of both environmental and health and higher education and scientific research ministries in Iraq

Conflict of Interest: The authors declare that they have no conflict of interest.

Funding: Self-funding

\section{References}

1- Grundy, SM. Hypertriglyceridemia, insulin resistance, and the metabolic syndrome. Am.J.cardiology. (1999). 83(9B) 25F-29F.

2- Ozder,IA. Lipid profile abnormalities seen in T2DM patients in primary healthcare in Turkey: a cross-sectional study. Ozder Lipids in Health and Disease2014.13:183-186

3- Nimmanapalli ,H.D. ; Kasi ,A.D. ; Devapatla, P.K. and Nuttakki,V. Lipid ratios, atherogenic coefficient and atherogenic index of plasma as parameters in assessing cardiovascular risk in type 2 diabetes mellitus . International Journal of Research in Medical Sciences. (2016). 4(7): 28632869.

4- Essiarab ,F.; Taki ,H. ; Lebrazi, H. ; Sabri ,M. and Saile, K. Usefulness of Lipid Ratios and Atherogenic Index of Plasma in Obese Moroccan Women with or without Metabolic Syndrome. Ethnicity \& Disease (2014). 24(2):207-212.
5- Dobiasova,M. ; Frohlich, J. ; sedova , M. ; cheung ,M.c. and Brown , B,G. Cholesterol esterification and atherogenic index of plasma correlate with lipoprotein size and findings on coronary angiography . J.Lipid Research. (2011). 52(3).56671.

6- Aronson, D. and Edelman, E.R. Coronary Artery Disease and Diabetes Mellitus. Cardiol clin. (2014). 32:439-455.

7- Chahil,T.JandGinsberg,H.N. Diabetic dyslipidemia.Endocrinol.metab.clin.North.Am (2006). 35:491-510.

8- Krauss , R.M. Lipids and Lipoproteins in Patients With Type 2 Diabetes. Diabetes Care . (2004). 27(6): 1496-1504.

9- Widlansky , M.E ; Gokce, N.;Keaney, J.F; J.A. The clinical implications of endothelial dysfunction.J.Am. coll.cardiol. (2003). 42(7):11491160 .

10- Halcox , J.P.; schenke ,W.H.; Zalos ， G ; Mincemoyer, M.and Prasad ,A. Prognostic value of coronary vascular endothelial dysfunction .circulation (2002). 106:653-658.

11- Verges,B.L. Dyslipidemia in diabetes mellitus , Review of the main lipoprotein abnormalities and their consequences in the development of atherogenesis.Diabetes and metabolism (1999). 25(3).32-40.

12- Tietz, N.W. Clinical Guide to Laboratory Tests. 3rd ed, W.B. saunders; co., Philadelphia, (1995). 22-23.

13- Jacobs, D.S.;Kasten, B.L.;De Mott,W.R. and Wolfson, W.L. In Laboratory and Test Handboook; Eds; Lexi-CompInc; Hudson(Cleveland), (1990). P.219.

14- Meigs ,J,B.; Mittleman,M.A.; Nathan,D.M.;Tofler, G.H.and singer,D.E. Hyperinsulinemia, Hyperglycemia, and Impaired Hemostasis. The Framingham Offspring Study. JAMA. (2000). 283(2):221-228.

15- Li,Z ; Huang,Qi; Li Sun,Li ; Bao,T and Zhe Dai. Atherogenic Index in Type 2 Diabetes and Its Relationship with Chronic Microvascular Complications. Hindawi International Journal of Endocrinology (2018).Volume, Article ID 1765835,9 pages. 